\title{
XXII. On the calculation of the distance of a shooting star eclipsed in the earth's shadow
}

\section{Archibald Smith Esq.}

To cite this article: Archibald Smith Esq. (1849) XXII. On the calculation of the distance of a shooting star eclipsed in the earth's shadow, Philosophical Magazine Series 3, 34:228, 179-182, DOI: $10.1080 / 14786444908646204$

To link to this article: http://dx.doi.org/10.1080/14786444908646204

曲 Published online: 30 Apr 2009.

Submit your article to this journal $[\pi$

Џll Article views: 3

Q View related articles $\asymp$ 
XXII. On the Calculation of the Dislance of a Shooting Star eclipsed in the Earth's Shadow. By Анснівацd SмIтн, Esq., of Lincoln's-Inn, Barrister-at-Larw, late Fellow of Trinity College, Cambridge*.

1. IN a paper in the Philosophical Magazine for February 1848, Sir John Lubbock has suggested that shooting stars may be small planetary bodies which shine by reflected light, and that their sudden disappearance may be occasioned by their immersion in the earth's shadow; and he has given a formula for calculating, on this hypothesis, the distance of a shooting star, at the moment of its disappearance, from a spectator on the earth's surface. The formula, however, as it is given in the paper in question, even when simplified by supposing the earth's shadow to be cylindrical instead of conical, is not well adapted for numerical calculation, and may repel some who would be inclined to pursue the investigation if the necessary calculations were less laborious.

The data assumed by Sir J. Lubbock are the zenith distances, and the difference of the azimuths of the sun and of the star at the moment of its disappearance. In repeating his calculations, I find that by introducing the angular distance between the shooting star and the sun, or rather the point opposite the sun, the formula is very much simplified, and thus the time requisite for calculating a distance does not exceed a very few minutes. As this is a point of some importance in furnishing a test for the theory, this communication may not be considered inappropriate to the Philosophical Magazine.

2. Let the centre of the earth be the origin of co-ordinates. Let the axis of $\mathbf{Z}$ be directed to the zenith, of $x$ to the north, and of $y$ to the east.

Let $x, y, x$ be the rectangular co-ordinates of the shooting star at the moment when it enters the earth's shadow.

$\rho$ its distance from the spectator.

$\zeta, \alpha$ its zenith distance and azimuth.

$a, b, c$ the co-ordinates of the vertex of earth's shadow.

$\mathrm{S}$ the length of the shadow $=\sqrt{a^{2}+b^{2}+c^{2}}$.

$\mathrm{Z}, \mathrm{A}$ the zenith distance and azimuth of the point in the heavens which is diametrically opposite to the sun.

$\phi$ the angular distance of the shooting star from the point opposite the sun.

$R$ semidiameter of the earth.

* Communicated by the Author.

$\mathrm{N} 2$ 
3. The equation to the cone of the earth's shadow is $\left\{x^{2}+y^{2}+z^{2}-\mathrm{R}^{2}\right\}\left\{s^{2}-\mathrm{R}^{2}\right\}=\left\{a x+b y+c z-\mathrm{R}^{2}\right\}^{2}$.

Also, since

it follows that

$$
\begin{array}{ll}
x=\rho \sin \zeta \cos \alpha & a=\mathrm{S} \sin Z \cos \mathrm{A} \\
y=\rho \sin \zeta \sin \alpha & \quad b=\mathrm{S} \sin Z \sin \mathrm{A} \\
z=\rho \cos \zeta+\mathrm{R} & c=\mathrm{S} \cos Z,
\end{array}
$$

$$
x^{2}+y^{2}+z^{2}=p^{2}+2 \mathrm{R} \rho \cos \zeta+\mathrm{R}^{2} \quad . \quad .
$$

$a x+b y+c z=S p\{\sin \zeta \sin Z \cos (A-a)+\cos \xi \cos Z\}+S R \cos Z$

$=$ (by spherical trigonometry) $\mathrm{S}_{\rho} \cos \phi+\mathrm{SR} \cos \mathrm{Z}$. .

It is the introduction of $\phi$ at this part of the process which so much simplifies the result.

Substituting in equation (1.) the values of $x^{2}+y^{2}+z^{2}$ and of $a x+b y+c x$, given by equations (2.) and (3.), and putting $q$ for $\frac{\mathrm{R}}{\mathrm{S}}, q$ being a small quantity, the average value of which is about $\cdot 0046$, we have

$$
\left.\begin{array}{c}
\rho^{2}\left\{\sin ^{2} \varphi-q^{2}\right\}+2 \mathrm{R} p\left\{\left(1-q^{2}\right) \cos \zeta-\cos \phi(\cos \mathrm{Z}-q)\right\} \\
-\mathrm{R}^{2}\{\cos \mathrm{Z}-q\}^{2}=0
\end{array}\right\}
$$

In this formula $\sin \phi$ may be considered as always greater than $q$, or $\phi$ greater than $16^{\prime}$; since, if smaller, it would be impossible to make any approximation to the distance of the shooting star. This equation, therefore, will have two roots; one positive, the other negative. The positive root is of course the only admissible solution. Solving the equation and making $R=1$, we obtain

$$
\begin{aligned}
p= & \sqrt{\left\{\frac{\left(1-q^{2}\right) \cos \zeta-\cos \phi(\cos Z-q)}{\sin ^{2} \phi-q^{2}}\right\}^{2}+\frac{(\cos Z-q)^{2}}{\sin ^{2} \phi-q^{2}}} \\
& -\frac{\left(1-q^{2}\right) \cos \zeta-\cos \phi(\cos Z-q)}{\sin ^{2} \phi-q^{2}} \text {. . . . . . . }
\end{aligned}
$$

The calculation of $\rho$ from this formula will be facilitated by the use of a subsidiary angle $\psi$, such that

we then have

$$
\cot \psi=\frac{\left(1-q^{2}\right) \cos \zeta-\cos \phi(\cos Z-q)}{\sqrt{\sin ^{2} \phi-q^{2}}(\cos Z-q)} ; . . .
$$

$$
\rho=\frac{\cos Z-q}{\sqrt{\sin ^{2} \phi-q^{2}}} \cdot \tan \frac{\psi}{2} \text {. . . . . }
$$

4. In almost all cases a sufficiently approximate result will 
be obtained by supposing the earth's shadow to be cylindrical instead of conical; the formula then become extremely simple. This is the same thing as supposing $q=0$; we then have

$$
\begin{aligned}
\rho^{2} \sin ^{2} \phi & +2 R_{\rho}(\cos \zeta-\cos \phi \cos Z)-R^{2} \cos ^{2} Z=0 . \\
\rho= & \left.\sqrt{\left\{\frac{\cos \zeta-\cos \phi \cos Z}{\sin ^{2} \phi}\right\}^{2}+\frac{\cos ^{2} Z}{\sin ^{2} \phi}}\right\} .
\end{aligned}
$$

and, as before, using a subsidiary angle $\psi$, but which is now determined by the equation

we have

$$
\cot \psi=\cos \zeta \sec Z \operatorname{cosec} \phi-\cot \phi, \quad \text {. . (10.) }
$$

$$
\rho=\cos Z \operatorname{cosec} \phi \tan \frac{\psi}{2} . \quad . \quad .
$$

These two formulæ furnish the means of calculating $p$ in a very few minutes with the aid of a table of logarithms and of natural tangents. Such tables (Hutton's, for instance, require to be opened seven times only.

5. The formula may be still further simplified by introducing, as one of the data, the angle contained between the great circles which pass through the shooting star and the sun, and the star and the zenith. As this angle may be directly obtained from a celestial globe without calculation, it may be worth while to exhibit the formulæ with this substitution. I believe, however, that although the formulæ thus become more simple in appearance, the calculation will be very little, if at all, facilitated thereby.

If we call this angle $B$, then by a well-known formula in spherical trigonometry we have

$$
\cos \zeta-\cos \varphi \cos Z=\sin \phi \sin Z \cos B ;
$$

and substituting this value in equation (8.), it becomes

$$
\rho^{2} \sin ^{2} \phi+2 R_{\rho} \sin \phi \sin \mathrm{Z} \cos \mathrm{B}-\cos ^{2} \mathrm{Z}=0 \text {. . }
$$

Solving this equation, we have

$$
\rho \frac{\sin \phi}{\sin Z}=\sqrt{\cos ^{2} B+\cot ^{2} Z}-\cos B ;
$$

and using, as before, a subsidiary angle $\psi$, such that

we have

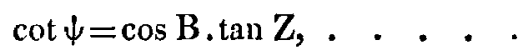

$$
\rho=\cos Z \operatorname{cosec} \phi \tan \frac{\psi}{2} \ldots . . .
$$


The calculation by means of equations (14.) and (15.) does not require a table of natural tangents. It requires the logarithmic tables to be opened six times.

6. For the use of persons who may wish to make the calculations without being able to follow the steps of this investigation, it may be desirable to give the result separately; it is this.

Let $\zeta$ be the zenith distance of the shooting star at the moment of disappearance, $Z$ the zenith distance of the point of the heavens diametrically opposite the sun, and $\phi$ the distance of this point from the shooting star. Find an angle $\psi$ such that

$$
\operatorname{cotan} \psi=\cos \zeta \sec \mathrm{Z} \operatorname{cosec} \varphi-\operatorname{cotan} \varphi .
$$

Then the distance $\rho$ of the shooting star from the spectator in terms of the earth's semidiameter as the unit is

$$
\rho=\cos Z \operatorname{cosec} \phi \tan \frac{1}{2} \psi \text {. }
$$

3 Stone Buildings, Lincoln's-Inn,

February 14, 1849.

XXIII. Remarks on the Weather during the Quarter ending December 31, 1848. By James Glaisher, Esq., of the Royal Obserzatory, Greenwich*.

THE meteorological returns for the past quarter furnished 1 to the Registrar-General have been received from stations spread over the country. The observations have been made, for the most part by experienced observers, upon an uniform plan. The following remarks are based upon observations which have been furnished either to myself or to the Registrar-General, and drawn up to accompany the meteorological tables published by the Registrar-General, all of which have been examined by myself, and reduced under my direction.

The weather during the period has been variable. 'The changes of temperature have benn frequent and great, there has been an nnusually large number of exhibitions of the aurora borealis, and the magnetic instruments have been greatly disturbed. The amount of electricity in the atmosphere has been small, many days together having passed without the instruments at Greenwich being affected.

From the 1st of October to the 10th the excess of temperature above the average of seven years was $6^{\circ} \cdot 6$; the greatest daily excess was $12^{\circ} \cdot 3$ on the 7 th. Between the 11 th and

* Communicated by the Author. 